\title{
Logic and reasoning in jokes
}

\author{
Graeme Ritchie
}

University of Aberdeen, United Kingdom

\begin{abstract}
It has often been remarked that jokes involve some form of distorted logic, but the details of this joke logic have not been fully explored. We offer a contribution to the methodology of this exploration by clarifying some abstract theoretical distinctions. Firstly, we separate two crucially different notions of "reasoning" which are relevant to joke comprehension: internal logic and audience inference. Internal logic is a system of logical rules, in the traditional sense, which define relationships within the fictional world of the joke, particularly the relation of consequence. Audience inference is a dynamic process which the recipient of a joke undertakes in order to make sense of it. Previous writings on the topic of logic in jokes seem to conflate these two very different concepts. Another distinction which is sometimes overlooked is between internal logic and other joke techniques with different functions, such as strategies for presenting information. We also consider whether the logic of jokes requires a qualitatively different inference mechanism from that of conventional logic, concluding that there is not yet any evidence to suggest this. Finally, we reflect on how we might go on to address the open question of what is possible as pseudo-logic within a joke.
\end{abstract}

Keywords: theory of humour; jokes; logic; inference; cognitive processing.

\section{Introduction}

There appears to be a consensus that jokes involve dubious logic (see references in Section 3 below). Nevertheless, theoretical understanding of the logical aspects of jokes is still relatively undeveloped. The aim of this article is to facilitate progress in this area, by arguing for some finer conceptual distinctions to be made. In particular: 
(i) a distinction should be made between the logic within the joke and the reasoning carried out by the recipient;

(ii) "pseudo-logic" in jokes should be distinguished from more general joke construction devices;

(iii) there is no evidence that joke pseudo-logic uses a different formal mechanism from conventional logic;

(iv) the question of how pseudo-logic might differ from correct reasoning is still open.

\section{Assumptions about text comprehension}

When describing the role of logic within jokes, it is necessary to make some assumptions about the overall framework of text comprehension, in order to have an environment within which the logical mechanisms operate. In this section, we make at least some of our assumptions explicit, in the hope that these assumptions are sufficiently general that the points made in later sections are compatible with a variety of models of language understanding and do not require a very specific theory of comprehension (see Jurafsky \& Martin 2000: ch. 18; Herman 2009 for some of the general issues).

We adopt the following broad characterisation of joke comprehension. The joke's audience perceives the content of the joke in stages, as it is presented (in a medium such as written text, sound, or pictures), and in response builds up what we will call a construal of the input perceived so far (cf. Brône \& Feyaerts 2004). This construal will involve abstract concepts, including many which are semantic (in the sense of being directly related to language), and usually some more general abstractions, with relational links between concepts.

Many jokes involve a fictional world, usually inhabited by fictional characters. The basic information about this storyworld (Herman 2009) will be part of the construal, but there may also be further content in the construal. In particular, there may be metalevel information about the content of the joke, for example if the audience is considering two possible interpretations of the perceived input. That is, where there is a fictional storyworld, we regard the "facts" about that storyworld to be object level information, allowing the possibility of higher information about the fiction; i.e. a metalevel.

We will use the term fiction-based joke to apply to any joke which operates by establishing a storyworld (either a supposed version of the real world, or something completely imaginary) in which there can be characters and events, even if the joke is not presented as a conventional narrative. Hence question-answer jokes starting with Did you hear about the ...? or posing a question about some real or fictional character, such as What's Bill Clinton's idea of safe sex?, will also count as fiction-based jokes.

There can be jokes which are not fiction-based, such as riddles involving word-play, where some of the above may not apply. The construal being built up may involve more abstract generalities, rather than a specific storyworld, but the audience will still have to make some cognitive effort to flesh out what has been presented.

We will interpret the term "logic" loosely, but in a way which captures the usual usage within more formal disciplines (see Section 5.1 for more discussion). We take a logical system to be a set of principles which specify the manipulation of propositional information (facts, hypotheses, assertions, etc.) about some particular "world" (real or imaginary, concrete or abstract). In particular, further information (consequences or conclusions) can be derived from 
existing information (assumptions or previous conclusions), thereby expanding the range of assertions deemed to be true in the "world".

The question of coherence within discourse is not simple (Brown \& Yule 1983; Toolan 2009), but we will assume that one important factor in the structuring of a narrative is logic, in the sense used here. This is not to say that all discourses adhere to sound and correct reasoning, as in the everyday sense of "being logical". Instead, we mean that the coherence of a story depends, at least in part, on links between items of information, so that there are reasons for why particular events happen or situations exist. The audience will (perhaps unconsciously) be aware of patterns of what always or typically follows from what, and will automatically use these patterns (rules) to connect the substance of the story together. Rules like this can formally constitute a logical system, even when they are empirically inaccurate (see Section 5.1 below).

We shall consider only the (fairly common) situation, in which the audience is aware, perhaps as a result of contextual clues, that a joke is being presented. This allows us to leave aside, for now, any issues regarding perlocutionary force, sincerity, mode of communication, etc. (cf. Raskin \& Attardo 1994; Dynel 2008).

\section{Internal logic vs. audience inference}

In the framework sketched in Section 2, there are two distinct notions of "reasoning" which are central to the operation of a joke, but which have not always been clearly distinguished.

The need for the first idea has often been mentioned in the literature on jokes. Freud (1966 [1905]) comments on the faulty reasoning embedded in various jokes. Attardo observes that "Several scholars have attempted to define the types of playful logic that seems to operate in humor" (Attardo 1994: 148), citing Aubouin (1948), Arieti (1967), Apter (1989) and Forabosco (1992). Attardo \& Raskin (1991: 304) note the relation of "paralogisms" in humour to the traditional "suspension of disbelief". Oring remarks that "The techniques of jokes are often paralogical, pseudological, or spurious", involving "...the drawing of conclusions from antecedent statements and facts even if those conclusions are not necessarily sound" (Oring 2011: 151). Ziv argues that jokes have a "local logic" - "local" in the sense that it is peculiar to jokes - occupying “...a middle position between logical and pathological thinking” (Ziv 1984: 98).

Most of these observations appear to postulate what we will call internal logic, since it operates within the joke. Any fictional world constructed for a joke (by the audience, reacting to the perceived input) conforms to its internal logic, even though the audience may be not fully aware of the details of this logic initially. That is, the notion of "consequence" within the joke world is defined by the rules of the internal logic.

The second type of reasoning, which we shall call audience inference, is a process at the centre of joke comprehension. The joke's audience carries this out incrementally, in the sense that the audience is gradually constructing (consciously or unconsciously) a construal of the joke, as information is received (Suls 1972; Ritchie 2004; Dynel 2012). This involves trying to assimilate information into the current version of the construal, using, where necessary, inference: a process of linking ideas, associating concepts, following known patterns, filling in defaults, etc. (Zwaan \& Singer 2003; Brown \& Yule 1983: ch. 7). This may require reconsideration of possible meanings for some portion of text (possibly using meta-linguistic reasoning), and often some inferences about what other (fictional) minds contain (theory of mind reasoning). 
There are, thus, two separate notions of "reasoning". Within the joke world, the events and situations are connected by an internal logic, which may be odd in some way (see Section 5 ), and which is not explicitly spelled out as a step-by-step process. On the other hand, the audience inference is about (rather than within) the joke world, is as valid as possible, and is a sequential process which attempts to build up a coherent construal. This does not claim that the audience will never make mistakes while interpreting a joke, as many jokes involve misleading the audience. However, such transient misinterpretation is a distinct phenomenon from the way that the internal structure of the joke is based on a flawed logic.

Some of the main contrasts between internal logic and audience inference are summarised in Table 1.

Table 1. Internal logic vs. audience inference

\begin{tabular}{|l|c|c|}
\hline & Internal logic & Audience inference \\
\hline Validity & Flawed & Correct \\
\hline Scope & Inside joke world & Meta-level \\
\hline Role & Licenses links in joke world & Builds construal \\
\hline Ordered in time & No & Yes \\
\hline
\end{tabular}

We can illustrate this using the traditional joke construct of Irish stupidity, in (1):

(1) Did you hear what happened to the Irish Sea Scouts? Their tent sank. (Tibballs 2000: 172)

In (1), the internal logic may be something like this:

Scouts put up tents. Therefore Sea Scouts will put up tents. Sea Scouts engage in activities on the sea. Therefore Sea Scouts will put up tents on the sea. A tent put up at sea will sink.

This is typical of the way that internal logic operates within a (fiction-based) joke. A character has a set of beliefs, some of which may be faulty, and the character may make some steps of reasoning, using those beliefs. Some of these steps may be faulty in some way. At some stage, this causes the character to act in a particular way (e.g. saying something), and the action is in some way inappropriate, in that either it violates (within the story world) some norms of behaviour or of commonsense, or it leads to some unfortunate consequences, or both. Often, the various steps of the logic are not made explicit, nor presented in logical order. It is not the case that the audience makes these faulty inferences; these logical slips are within the joke, often perpetrated by a character within the joke narrative.

In contrast, the audience inference involves unpicking the information, perhaps like this:

The tent sank, so it must have been on a body of water. This was because it belonged to a searelated group. They put up a tent because they are Scouts, and Scouts put up tents.

The inference is an attempt to make sense of the information supplied, often working backwards from the punch line, figuring out what may be going on in the fictional world of the joke, in order to construct a coherent construal, and postulating faulty logic as necessary. As Ziv observes: "The cognitive process aims at understanding and 'solving' an incongruity, thus restoring balance..." (Ziv 1984: 96). 
The distinction between internal logic and audience inference matters for a number of reasons. If we are trying to elaborate the details of what previous authors have referred to as "pseudo-logic", we should concentrate on the internal logic of the joke, without being distracted by the possible cognitive efforts the audience might make to understand the joke. Conversely, in mapping out the comprehension process, we should not confuse it with faulty logic which joke characters may adopt (although the audience inference clearly is about the joke-internal logic). These notions have substantially different characteristics, particularly whether or not each is a process.

Past discussions of "logic" in jokes sometimes seem to conflate these two concepts. Some of Ziv's remarks about "local logic" apparently describe what we term "internal logic", but he sometimes seems to imply that the audience uses the flawed logic:

“...to resolve incongruities, one can either use logic or employ a different mode of thinking. The particular way of approaching incongruity in humor is what I call local logic ..." (Ziv 1984: 81). "We are accustomed to logical thinking, and all of a sudden it does not work. Failing to solve a joke's incongruity by logic, we try the thought mode of local logic." (Ziv 1984: 96)

A similar observation could be made about the General Theory of Verbal Humor (GTVH) (Attardo \& Raskin 1991), which includes the Logical Mechanism (LM), described as "paralogical" (Hempelmann \& Attardo 2011: 147) and "a distorted, playful logic, that does not hold outside the world of the joke" (Attardo 1994: 226). These descriptions seem to have the LM describing aspects of the joke's meaning, thus involving logical connections within the joke. On the other hand, the LM is a "dynamic cognitive process" (Hempelmann 2004: 383), and "the LM is the resolution itself, not just an enabling mechanism thereof ...the resolution is a dynamic phenomenon, the enabling mechanism is a static component of the text" (Attardo 1997: 409). However, both in the latter article (Attardo 1997: 410) and elsewhere (Attardo et al. 2002; Hempelmann \& Attardo 2011), illustrative examples of LM seem to be statements of semantic or other relationships within jokes rather than processes.

\section{Internal logic vs. joke mechanisms}

The notion of internal logic that we are setting out here is relatively narrow. It is solely concerned with the notions of consequence and causation within the world of the joke: why a certain situation arises or a particular event occurs within that world, where the answer to "why" is in the mind of a joke character or the joke narrator, not in some wider purpose or function of the joke (such as releasing repressed emotions). There are many other noteworthy aspects of a joke, apart from the internal logic. There are presentation devices: a joke often supplies information in a particular way, withholding crucial details to mislead the audience. A joke may employ ambiguity in order to bring incompatible meanings together, or there may be some form of blending of ideas. A full list of "joke mechanisms" would depend on how widely that term is defined.

Some previous discussions of joke devices cover both our narrower notion of internal logic and some of these other (important, but different) facets of joke structure, without drawing attention to this distinction. Our rationale for taking the narrower remit is that it is desirable, in mapping out the workings of jokes, to draw distinctions between mechanisms which appear to have separate functions or to be related to different facets of joke 
comprehension. Although it is often helpful to seek generalisations, too much grouping together of quite different aspects of jokes can obscure important patterns and relationships.

The seminal discussion of joke-work by Freud (1966[1905]) takes this more wideranging view. We are arguing not that Freud was necessarily mistaken in his cataloguing of techniques, but rather that, in assessing any one of Freud's techniques, we have to ask not only "is this an accurate assessment of what is happening in these jokes?" but also "which aspect of joke structure is it describing, and to which component of a formal model might it contribute?".

Ziv (1984: 92) mentions very briefly some tricks which occur in his "local logic", one or two of which seem to be devices of internal logic, such as internal contradiction. However, others seem to be more about a broader view of the events which occur within a joke, rather than the underlying logic, such as role change (where two participants act out each other's normal roles). As with Freud, the question is not whether these intuitive insights are persuasive, but where within a model of joke comprehension they fit best.

There is a similar ambiguity about the "LM" of the GTVH (Section 3 earlier). It is far from clear that all LMs refer to comparable aspects of the joke. Although a few illustrative LMs are mentioned by Attardo \& Raskin (1991) and Hempelmann \& Attardo (2011), a much fuller catalogue is given by Attardo et al. (2002) (revising a list proposed by Paolillo (1998)). It is intuitively clear that each specific LM describes some salient aspect of the joke, but it is far from clear that these different LM examples illustrate the same aspect. Some LMs describe situations within the world of the joke, and hence are like our internal logic; these include ignoring the obvious and reasoning from false premises (most LMs are given informal labels rather than formal definitions). On the other hand, some LMs are about the devices used to present information in order to manipulate the audience's reasoning about the joke - the metaforms of Attardo et al. (2002). These include the garden-path LM (where the early part of a joke leads the audience to expect one interpretation, but the punch line forces a different interpretation to the fore) and the consequence LM in which "a situation representing a consequence of some event is represented, leaving prior series of events to be inferred" (Paolillo 1998: 271). Some LMs are concerned with relationships between the two scripts which are posited to underlie the joke; that is, they concern the overall conceptual structure of the joke. Hempelmann \& Attardo (2011: 127) emphasise that the use of inference in LMs is a hypothesis, not a defining feature. We are not arguing that these observations about patterns within jokes are mistaken, but rather that the notion of LM is extremely heterogeneous, covering several facets of joke mechanisms, whereas our notion of internal logic is much narrower.

If we delineate internal logic as a specific component of joke structure, this allows us to investigate its nature in more detail, without having to also describe other facets, such as presentation strategies, as if they were naturally part of the same component.

\section{The nature of pseudo-logic}

\subsection{Is there a formally distinct mechanism?}

Some authors strongly suggest that the faulty logic which is central to a joke is based on a different kind of logic; that is, a mechanism which is qualitatively different from conventional logical reasoning. The widespread use of terms such as "pseudo-logic" or "paralogic" hints at a different specialised reasoning device. In addition to the quotations in Section 3 above, Ziv 
says that to enjoy humour "one has to ...take momentary leave of Aristotelian logic" (Ziv 1984: 77), and Attardo \& Raskin remark that "the use of paralogisms instead of correct syllogisms in humor is quite permissible" (Attardo \& Raskin 1991: 304).

The examples usually adduced to support the need for "pseudo-logic" simply demonstrate that reasoning within the joke world has arrived at a conclusion which is somehow strange. Typical types of strange outcomes are: being empirically incorrect; being less likely than other possible conclusions; resulting in a situation which violates norms (social, cultural, etc.). None of these indicate that the underlying reasoning mechanism is itself faulty - it may just be that the agent performing the reasoning is working from slightly different facts (assumptions, estimations of probabilities, etc.). A completely sound (and conventional) reasoning process can arrive at dubious conclusions if it starts from incorrect information.

It might seem that there are plenty of examples of jokes which depend on a particular flawed step in the reasoning, not on sound reasoning from incorrect premises, and that such humour suggests the need for a non-standard logical mechanism. To examine this properly, it is necessary to make a brief digression into what is meant by "logic", within philosophy, mathematics, and similar disciplines.

The main distinction to be emphasised is between the formal inferential mechanism of the logical system, and the substance of the statements upon which that mechanism operates. Different types of logical system have different formal inference methods. Once a logical system (with its mechanisms) is selected, notationally correct statements can be used to describe a particular (real or fictional) world. Inferences about that world can be made by having the mechanisms of the chosen system operate upon those statements. The truth (with respect to the particular world) of the statements produced will depend on the truth of what is fed into the mechanisms. There is a further subtlety. What might be, informally and intuitively, seen as "inference rules" or "possible steps in reasoning" can be represented within the logical notation as statements. If we wish to say that proposition $D$ always follows whenever $C$ is known, this can be written in the logic as if $C$ then $D$ (sometimes known as a material implication). The logical inference mechanism will normally, starting from two statements $C$ and if $C$ then $D$, infer that $D$ is also the case (a very common inference step, sometimes known as modus ponens). Thus if we start from the statements "Jock is a Scotsman" and "if Jock is a Scotsman, then Jock is cautious with his money", the natural conclusion is "Jock is cautious with his money". In this way, statements that feel rather like "logical rules" can be written to express connections between ideas within the world. These will then (assuming the underlying logical system has the appropriate inferential mechanism) lead to the connections being made. The phrase "inference rule" is sometimes used in discussing logical mechanisms, but this is ambiguous between the various parts of the inferential mechanism (e.g. modus ponens) or the if ...then kind of statement.

The reason for introducing these technicalities is to pose the question: if joke logic is non-standard, which formal aspects of standard logic are altered? Are different inferential mechanisms required?

The default assumption would be that there are no inference mechanisms which are peculiar to jokes. Although humorous phenomena are interestingly different from nonhumorous ones, we should be reluctant to multiply the number of constructs we postulate that are used only in humour. Any proponent of the more radical position - that joke logic requires inferential mechanisms different formally from ordinary logic - bears the onus of proof. So far, no real evidence for the existence of non-standard inferential mechanisms has been put forward. 
The faulty reasoning central to example (1) earlier could be achieved with a conventional inference mechanism. Example (2), below, illustrates both that the fault may simply be a mismatch with reality (i.e. incorrect assumptions), and also that the error may be comparatively small.

(2) Two rabbits and a hedgehog were sitting talking by the side of a busy road. The hedgehog was keen to see what was on the other side of the road but was frightened of attempting the journey for fear of being run over by a car. The rabbits gave him a piece of advice: 'If there's a vehicle coming,' they said,'look it right between the headlights, curl up in a tight ball, let it pass over the top and away you go. It's as simple as that.' A few minutes later, the first rabbit tried to cross the road. Half-way across, he saw a car approaching. Lining himself up directly between the car's headlights, he curled up in a ball and allowed the wheels to pass either side of him. Then he scampered to the grass verge on the other side. Shortly afterwards, the second rabbit crossed the road. He was almost at the other side when he saw the headlights of a huge lorry. Lining himself up directly between the headlights, he curled up in a ball and allowed the wheels to pass either side of him. Then he too scampered to the grass verge. Five minutes later, the hedgehog finally plucked up the courage to try and cross the road. He had only gone a few yards when he saw headlights approaching. He lined himself up directly between the headlights, curled up in a ball and splat! He was run over. One rabbit turned to the other and said, 'That was bad luck. How many Reliant Robins do you get on this road?'

(Tibballs 2000: No. 193, 29)

(To interpret this slightly dated British joke, it is necessary to know that a "Reliant Robin" was a well-known UK model of three-wheeled car, with its single front wheel centrally mounted.)

In this tale, the animals operate with a near-correct statement about motor vehicles: "in the centre of a vehicle, there are no wheels to squash a small animal", instead of a more accurate - but very similar - statement, "in the centre of a conventionally four-wheeled vehicle ...". There is no indication that the scenario depicted in the joke requires the reasoning mechanism to be faulty. This example is not presented here as a critical counter-example, but merely as an illustration that at least some jokes do not appear to rely on a formally nonstandard inferential system. Although it is a plausible hypothesis that a joke will always contain an instance of reasoning that has gone wrong in some way, it is harder to make the case that this always involves a formally flawed reasoning mechanism. This still leaves open the possibility that there are some jokes which require unconventional reasoning methods, but this remains to be shown.

It is hard to be sure about the position of the GTVH's Logical Mechanisms (LM) on this issue. Most presentations of LMs stress that they involve "local logic" or "pseudo-logic" (see quotations in Section 3 above), so LMs are non-standard in some way. On the other hand, "... there is no ground to the claim that Logical Mechanisms are specific to humor ...we claim that none of the mechanisms of humor is unique per se to humor" (Hempelmann \& Attardo 2011: 126), and lists of LMs (e.g. Attardo et al. 2002: 18) include non-humorous items such as juxtaposition and analogy. The GTVH writings do not make the formal distinction between inference mechanism and the content of (possibly implicative) statements, and indeed do not explain how "logic" in the normal sense of the word is involved in LMs. It is, therefore, difficult to pose the question about inference mechanisms within the GTVH. The natural assumption is that the claim quoted from Hempelmann \& Attardo (ibid.) also covers inference 
mechanisms (although, as noted earlier, not all LMs necessarily involve inference). This leaves open exactly where the faultiness ("pseudo-logic") lies within LMs.

\subsection{How faulty can the logic be?}

Even if we accept that the flawed logic within a joke could be viewed, formally, as the use of inaccurate statements, that still leaves the question: what sort of inaccurate statements can be used in this way (i.e. to create humour)? Is there any principled limit, or can any inaccuracy whatsoever be pressed into service?

Brône \& Feyaerts (2004: 363-364) argue for one particular mechanism: confusion between a typical instance of a concept and a peripheral (untypical) instance, in the sense of Rosch (1973). This could, for example, be applied to (2) above, where the core and peripheral concepts of road vehicle are at the heart of the joke. It remains to be seen whether this single form of mistaken reasoning can account for a wide range of jokes.

Although it would be convenient if there were no limits on what sorts of inaccurate statements, including implication statements, could be used for humorous effect, this position seems highly implausible. Viable joke logic cannot be created simply by constructing arbitrarily faulty implication statements if $C$ then $D$ where $C$ and $D$ are randomly and independently chosen, such as "if the protagonist is Irish, then the situation takes place on Mars". Intuitively, it seems that the flawed statements, whether simple facts or implication statements, must have a close resemblance to a valid line of reasoning, differing only in small, systematic respects, so that the flawed statements are relatively similar to non-flawed versions (cf. example (2)).

There is one rather mundane consideration which may account for this requirement that the internal logic cannot be too outlandish: it must be relatively straightforward for the audience, via inference, to work out the nature of the internal logic. The joke text typically does not explicitly present the steps of the internal logic, but merely gives some clues as to its effects, and hence the audience inference involves some detective work to guess at the flawed reasoning within the joke. A logical step which was wholly arbitrary and totally unrelated to any of the audience's knowledge (either of reality or of typical storyworlds) would probably be too difficult to figure out. This is one advantage of distinguishing, in research, between internal logic and audience inference: the nature of the former is constrained by what can be determined by the latter.

To determine just what deviations from accuracy, or what notion of "similarity" to sound logic is appropriate, may well require a detailed examination of the logical flaws within a wide variety of jokes, to see what generalisations (if any) can be made; such a study is beyond the scope of the current paper. Davies (2004: 379) is strongly critical of the construction of taxonomies of logical mechanisms used in jokes, demanding rhetorically "What general conclusions can you draw...?". The response to this scepticism is that it is hard to know what generalisations will emerge until one has examined the material. The aim of such an investigation would not be to create a taxonomy for its own sake, but to make a step towards determining exactly what kind of distorted reasoning can function as a component of jokes. Crucial to this line of argument is the conjecture (see above) that the internal logic of jokes cannot be arbitrarily and randomly ill-formed, since setting out to catalogue every conceivable form of incorrect logical statement would be futile. The aim would be to delineate some notion of "mildly and systematically incorrect" which is suited to yielding humorous effects (and, as noted above, allows detection by the audience's inference). 
Inspecting some previous analyses, such as those discussed in Section 4, gives a hint at how large an undertaking this could be, and how complicated it could be to intuitively extract the logical core of many jokes.

\section{Summary}

The idea that there is a peculiar logic at the core of jokes is often stated. It is appropriate, therefore, to investigate in more detail what such a "pseudo-logic" might consist of. We have argued these main points:

(i) It is important to distinguish the flawed logical connections that underlie the events and situations in the fictional world of the joke from the process which the audience of the joke goes through in making sense of the joke.

(ii) Some previous discussions of joke mechanisms include what seem to be instances of joke-internal flawed logic, but grouped together with other, formally and functionally different abstract aspects of jokes. Separating these different aspects will help in elucidating the logic within jokes.

(iii) Although joke logic is sometimes referred to as if it were a different formal mechanism from conventional logic, there is not yet any evidence to support this, as the incorrectness of a line of reasoning does not indicate a different reasoning mechanism.

(iv) What degree of faultiness is viable within the internal logic is still very much an open question.

This discussion should facilitate further understanding of the inner workings of jokes. Two further lines of enquiry which would be worth pursuing are to examine more examples to find what generalisations (if any) can be made about the ways in which internal joke logic can be faulty (Section 5.2), and to see whether the distinctions made here help to clarify the concept of "incongruity-resolution" (Martin 2007: 64-75).

\section{References}

Apter, M. J. (1989). Reversal Theory: Motivation, Emotion and Personality. New York \& London: Routledge.

Arieti, S. (1967). The Intrapsychic Self. New York \& London: Basic Books.

Attardo, S. (1994). Linguistic Theories of Humor. Berlin: Mouton de Gruyter.

Attardo, S. (1997). 'The semantic foundations of cognitive theories of humor'. Humor: International Journal of Humor Research 4 (10), pp. 395-420.

Attardo, S., Hempelmann, C. \& Di Maio, S. (2002). 'Script oppositions and logical mechanisms: Modeling incongruities and their resolutions'. Humor: International Journal of Humor Research 15 (1), pp. 3-46.

Attardo, S. \& Raskin, V. (1991). 'Script theory revis(it)ed: Joke similarity and joke representation model'. Humor: International Journal of Humor Research 4 (3), pp. 293347.

Aubouin, E. (1948). Technique et Psychologie du Comique. Marseilles: OFEP.

Brône, G. \& Feyaerts, K. (2004). 'Assessing the SSTH and GTVH: A view from cognitive linguistics'. Humor: International Journal of Humor Research 17 (4), pp. 361-372. 
Brown, G. \& Yule, G. (1983). Discourse Analysis. Cambridge: Cambridge University Press.

Davies, C. (2004). 'Victor Raskin on jokes'. Humor: International Journal of Humor Research 17 (4), pp. 373-380.

Dynel, M. (2008). 'There is method in the humorous speaker's madness: Humour and Grice's model', in Dynel, M. (ed.), Łódź Papers in Pragmatics: Volume 4.1, Łódź, Poland: Łódź University Press, pp. 159-185.

Dynel, M. (2012). 'Garden paths, red lights and crossroads: On finding our way to understanding the cognitive mechanisms underlying jokes'. Israeli Journal of Humor Research 1 (1), pp. 6-28.

Forabosco, G. (1992). 'Cognitive aspects of the humor process: the concept of incongruity'. Humor: International Journal of Humor Research 5 (1/2), pp. 45-68.

Freud, S. (1966[1905]). Jokes and their Relation to the Unconscious. Translated by Strachey, J. London: Routledge \& Kegan Paul.

Hempelmann, C. (2004). 'Script opposition and logical mechanism in punning'. Humor: International Journal of Humor Research 17 (4), pp. 381-392.

Hempelmann, C. \& Attardo, S. (2011). 'Resolutions and their incongruities: Further thoughts on logical mechanisms'. Humor: International Journal of Humor Research 24 (2), pp. $125-149$.

Herman, D. (2009). 'Cognitive Narratology', in Hühn, P., Pier, J., Schmid, W. \& Schönert, J. (eds.), Handbook of Narratology, Berlin: Walter de Gruyter, pp. 30-43.

Jurafsky, D. \& Martin, J. H. (2000). Speech and Language Processing: An Introduction to Natural Language Processing, Computational Linguistics and Speech Recognition. New Jersey: Prentice-Hall.

Martin, R. A. (2007). The Psychology of Humor: An Integrative Approach. London: Elsevier Academic Press.

Oring, E. (2011). 'Still further thoughts on logical mechanisms: A response to Christian F. Hempelmann and Salvatore Attardo'. Humor: International Journal of Humor Research 24 (2), pp. 151-158.

Paolillo, J. C. (1998). 'Gary Larson's Far Side: Nonsense? Nonsense!'. Humor: International Journal of Humor Research 3, pp. 261-290.

Raskin, V. \& Attardo, S. (1994). 'Non-literalness and non-bona-fide in language: Approaches to formal and computational treatments of humor'. Pragmatics and Cognition 2 (1), pp. 31-69.

Ritchie, G. (2004). The Linguistic Analysis of Jokes. London: Routledge.

Rosch, E. (1973). 'On the internal structure of perceptual and semantic categories', in Moore, T. E. (ed.), Cognitive Development and the Acquisition of Language, New York: Academic Press, pp. 111-144.

Suls, J. M. (1972). 'A two-stage model for the appreciation of jokes and cartoons: An information-processing analysis', in Goldstein, J. H. \& McGhee, P. E. (eds.), The Psychology of Humor, New York: Academic Press, pp. 81-100.

Tibballs, G. (ed.) (2000), The Mammoth Book of Jokes. London: Constable \& Robinson.

Toolan, M. (2009). 'Coherence', in Hühn, P., Pier, J., Schmid, W. \& Schönert, J. (eds.), Handbook of Narratology, Berlin: Walter de Gruyter, pp. 44-62.

Ziv, A. (1984) Personality and Sense of Humor. New York: Springer.

Zwaan, R. A. \& Singer, M. (2003). 'Text comprehension', in Graesser, A. C., Gernsbacher, M. A. \& Goldman, S. R. (eds.), Handbook of Discourse Processes, Mahwah, NJ: Lawrence Erlbaum Associates, pp. 83-121. 